\title{
Light induced stimulation and delay of cardiac activity
}

\author{
Boris Hofmann $^{\text {a\#, }}$, Vanessa Maybeck ${ }^{\text {a\#\# }}$, Stefan Eick ${ }^{a}$, Simone Meffert ${ }^{\mathrm{a}}$, Sven Ingebrandt ${ }^{\mathrm{b}}$, Philip Wood ${ }^{\mathrm{c}}$, \\ Ernst Bamberg ${ }^{\mathrm{c}}$ and Andreas Offenhäusser*a \\ Received (in $X X X, X X X)$ Xth $X X X X X X X X X 200 X$, Accepted $X$ th $X X X X X X X X X 200 X$ \\ ${ }_{5}$ First published on the web $X$ th $X X X X X X X X X 200 X$ \\ DOI: $10.1039 / b 000000 x$
}

This report shows the combination of light activatable ion channels and micro electrode array (MEA) technology for bidirectionally interfacing cells. HL-1 cultures, a mouse derived cardiomyocyte-like cell line, transfected with channelrhodopsin were stimulated with a microscope coupled $473 \mathrm{~nm}$ laser and recorded ${ }_{10}$ with custom built 64 electrode MEAs. Channelrhodopsin induced depolarization of the cell can evoke action potentials (APs) in single cells. Spreading of the AP over the cell layer can then be measured with good spatiotemporal resolution using MEA recordings. The possibility for light induced pacemaker switching in cultures was shown. Furthermore, the suppression of APs can also be achieved with the laser. Possible applications include cell analysis, e.g pacemaker interference or induced pacemaker switching, and medical ${ }_{15}$ applications such as a combined cardiac pacemaker and defibrillator triggered by light. Since current prosthesis research focuses on bidirectionality, this system may be applied to any electrogenic cell, including neurons or muscles, to advance this field.

\section{Introduction}

Electrogenic cell networks, in the form of nerves and muscles, 20 are at the heart of animal physiology and behavior. To adjust or repair signaling in these systems, it is often necessary not only to provide signals to the network, but also to simultaneously record network activity. Applications for treatment of several kinds of diseases or disorders such as loss 25 of sensory perception [1, 2, 3] central nervous system disorders or loss of limbs $[4,5,6]$ would all benefit from bidirectional communication with electrogenic networks of cells.

Micro electrode arrays (MEAs) have been established as an 30 electrical recording system that is non-invasive for electrogenic cells [7]. MEA recording does not disturb cell signaling and generates scar tissue only upon initial placement. Multi-site analysis of tissue is one of the most common applications for MEAs in vitro. Application of ${ }_{35}$ MEAs to record heart tissue $[8,9]$ as well as neuronal network behavior $[10,11]$ is commonly done in various types of in vitro and in vivo preparations. Advantages are the good spatial resolution in combination with a high temporal resolution. This combination is seldom found together in other 40 commercially available monitoring techniques, and allows the analysis of the origin and propagation of cellular signals, the amount of action potentials fired, or even changes in signal shape. Custom made or prototype CMOS-MEAs achieve higher point to point resolution, but require slower sampling 45 rates or use of only a fraction of the channels to monitor an area as large as the conventional MEA [12].

To stimulate action potentials in the cell network, electrical stimulation via micro electrodes is the most common technique used. In vitro experiments with MEAs showed that 50 current or voltage controlled stimulation $[13,2,14,15]$ can be used for reliable depolarization of electrogenic cells and in vivo applications are already employed. Electrode based implants not only restore the senses $[1,16,17,18]$, but are also utilized for treatment of Parkinson disease during deep ${ }_{55}$ brain stimulation [19]. Electrical stimulation is also used to generate models of disease in vitro $[20,21]$. However, the 
most common application of electrical microstimulation is probably as cardiac pacemakers [22, 23] and more recently also implantable cardioverter-defibrillators [24].

Nevertheless, all applications of electrical stimulation have to 5 deal with several drawbacks, like scar formation around the electrode [25] or permeabilization of the cells during stimulation. Scar formation requires use of higher stimulation voltages, which may exceed the electrochemical window of the electrodes and are therefore not suitable. Of even greater 10 concern is the fact that electrical stimulation can permeabilize the cell. Electropermeabilization of the cells can trigger action potentials, but at the same time damages the cell, preventing the desired long term interfacing [26]. Furthermore, the number and identities of the electrically microstimulated 15 neurons, as well as the number of action potentials evoked, are difficult to determine. This is particularly problematic when functionally different cells lie in close spatial proximity. For these reasons we seek reliable alternatives to electrode stimulation for artifact-free, controllable, and specific cell 20 stimulation [27, 28]. Promising results were achieved using light triggerable ion channels that depolarize a cell and subsequently cause action potentials [29, 30, 31]. Nagel et al. found and established channelrhodopsin-2 (Ch2), a directly blue-light-gated cation channel, which binds retinal to form 25 the ChR2 complex [32, 33]. Stimulation can be applied rapidly and non-invasively to a limited subset of genetically defined cells reproducibly [34, 35]. Though genetic engineering can be tricky and requires sophisticated techniques for high efficiency or influence free transfection, 30 two main approaches exist in vivo; viral transfection [36], with high efficiencies but increased biological safety risk, or electrofection, with high efficiencies but limited application regions [37, 38, 39]. Several tissues have been successfully transfected with $\mathrm{Ch} 2$ allowing optical stimulation including 35 retina [40], somatosensory neurons [41], and cortex [42] and devices for light delivery have been designed [43]. Although this new tool in electrophysiology provides plenty of innovative ways of interfacing electrogenic cells, several drawbacks still have to be faced before implementation of 40 such concepts can be progressed. Among these is the need for a deeper understanding of cell network interactions during ChR2 activation. Despite behavioral studies using ChR2 in vivo, quantitation of cellular responses has relied largely on patch clamp or calcium imaging techniques. These methods 45 limit the number of cells that can be investigated at one time or have slow response times, respectively. Extracellular recording using chip technology, including MEAs, overcomes these limitations.

In this work we have combined the leading technologies of 50 optical stimulation and MEA recording to address the potential use of ChR2 in yet another role currently filled by electrode stimulation, the modulation of cardiac function. We used the cell line HL-1, which forms a confluent layer of cardiomyocyte-like cells, connected by gap junctions [38] to 55 model ChR2/MEA applications in the heart. HL-1 have already been used as a system to test the ability to introduce a dominant pacemaker into a cardiac tissue using mixed cultures

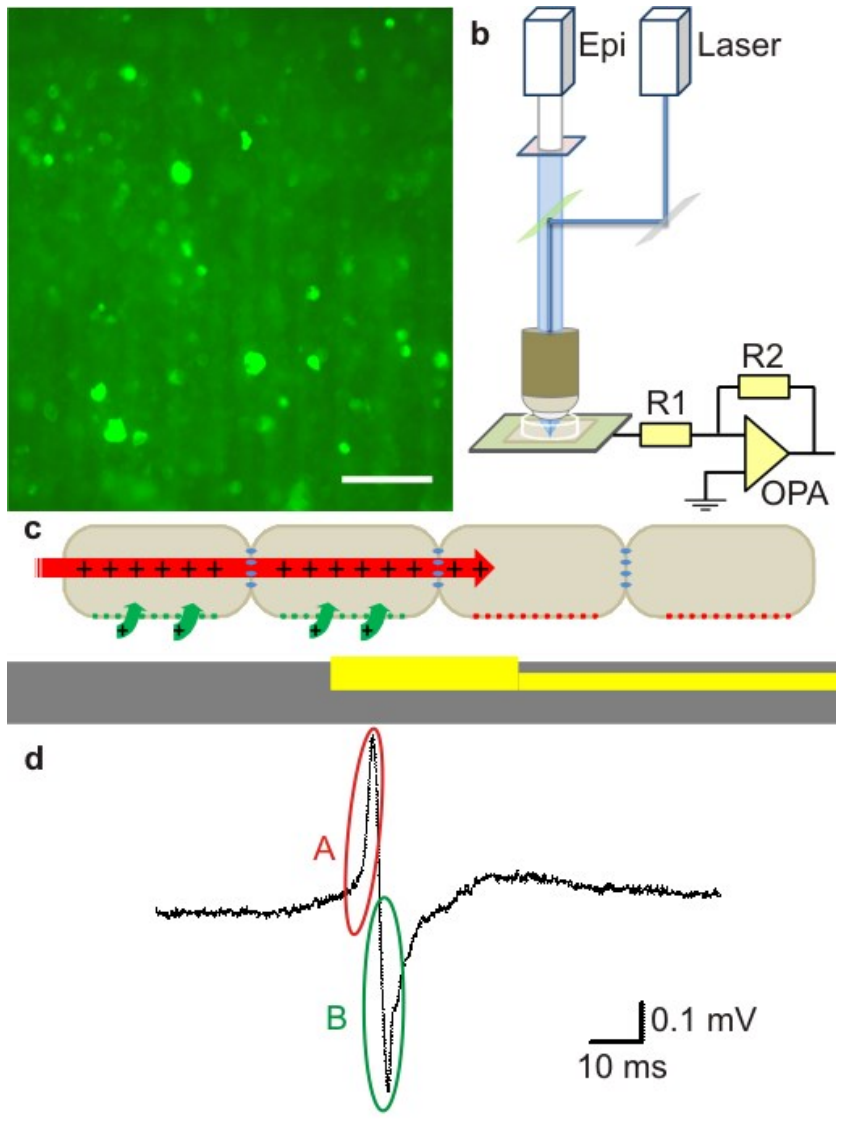

Experimental setup

60 A: $\quad$ Fluorescence image of electrofected $\mathrm{HL}-1$ cells. The different expression levels of the eYFP fused channelrhodopsin can be seen. Even though the transfection rate is very high, only the brightest cells were targeted for stimulation.

B: $\quad$ Schematic drawing of the stimulation setup. The upright 65 microscope can be used with any kind of transparent or non-transparent substrate including our MEA system. The laser is coupled to the microscope via a scanning device and a dichroic mirror and subsequently focused onto the substrate by the microscope optics. Epifluorescent as well as brightfield- or DIC-illumination can be combined with the laser 70 stimulation.

C: $\quad$ Example action potential recording from $\mathrm{HL}-1$ on extracellular MEA. Capacative coupling of depolarization through gap junctions is circled in orange. In green, the fast influx of positive ions from the gap between the cell and electrode when voltage 75 gated channels open generates a negative signal.

D: Schematic of ion flows through the sheet of cells at the front of the AP propagation wave. Depolarization propagates laterally (orange) through gap junctions (blue) to trigger an AP in the 80 neighboring cell. When the cell reaches threshold, voltage gated channels open and charge flows from the gap between cell and electrode into the cell (green).

of HL-1 and embryonic stem cell derived cardiomyocytes 85 [44]. Furthermore, the cell line has been regularly depolarized to model excess calcium in the heart of atrial fibrillation patients and conduct drug and genetic studies [21,20]. 


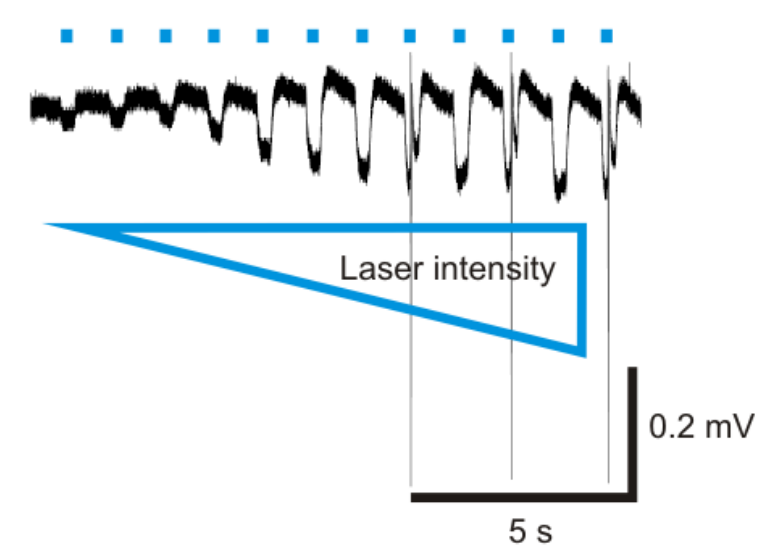

Figure 2: Optical stimulation

A: $\quad$ A single channel recording close to the artificial pacemaker 5 during $1 \mathrm{~Hz}$ photostimulation. Laser flashes (blue bars) of increasing intensity elicit first sub-threshold depolarization and at higher intensity trigger APs. Laser intensity was ramped from 5-40 mW.

The use of ChR2 with micro electrode recordings has enabled us to interface transiently transfected HL-1 cells 10 bidirectionally. The resulting potential use of light triggered channels for cardiac applications is shown. We demonstrate the ability of ChR2 to induce pacemaker switching in HL-1 cultures and alter cardiac activity. It is possible to increase as well as decrease beat frequency using light stimulation, 15 bringing to mind the potential of a combined pacemaker and defibrillator system. As the cardiac pacemaker was a forerunner to deep brain stimulation, so can advancements in optical control of cardiac tissue lead to insights for modulation of other electrogenic networks such as the brain.

\section{Results}

\section{Genetic engineering}

After electrofection, roughly $50 \%$ of the cells were positive for the expressed protein as determined by fluorescence of the 25 fusion protein. The transfection was analyzed using a standard eYFP filter set in combination with the fluorescence microscope. As Ch2 is fused to eYFP, the expression level should be directly linked to the fluorescence and only cells with a high fluorescence level were used for experiments 30 (Fig.1a), even though fluorescence does not give information about the amount of ChR2 formed from $\mathrm{Ch} 2$ or the amount of ChR2 inserted into the membrane. After seeding, the cells formed a confluent layer after 2-4 days on our custom made MEAs. Formation of the confluent layer was monitored 35 microscopically. Cultures began to spontaneously generate action potentials approximately one day after confluency, but were electrically connected via gap junctions before this. The intrinsic electrical activity was monitored via extracellular recordings, to ensure an electrically connected cell layer 40 before starting the experiments independent of visible

contractions. The extracellular signal highly depends on the origin of the ions contributing to the depolarization of the cells. Influx of sodium via the gap junctions that connect the cells causes no change of the ionic composition of the cleft, ${ }_{45}$ but changes the ionic distribution in the cleft. This is coupled to the electrode capacitively as a positive peak. Once the cell is depolarized above threshold, ion channels open and the sodium influx from the cleft changes the ionic composition above the electrode. The cleft is depleted from positive ions, 50 which can be seen as a negative peak.

\section{Light stimulation}

After choosing an appropriate transfected cell, light stimulation was performed using the combined recording and ${ }_{55}$ stimulation setup (a schematic drawing can be seen in Fig. 1b). Stimulation was performed with a laser spot diameter of approximately $10 \mu \mathrm{m}$. Recordings were done extracellularly with the MEA. With varying laser power, the depolarization of the ChR2 positive cell could be controlled and 60 subsequently action potentials were generated. On MEA electrodes in close proximity, the effect of laser intensity on the depolarization level could be measured as shown in Fig. 2. Light intensity variations between 5 and $40 \%$ of the laser were investigated and are indicated by the blue triangle. While ${ }_{65}$ lower intensities only evoked cellular depolarization (the direction of the signal is opposite the intracellular potential because it is recorded extracellularly at the cleft between cell and electrode), higher intensities evoked action potentials The measured intrinsic AP (see Fig.3a and 3d) differs from the 70 light evoked AP (Fig. 3 b and 3e.). Subtracted from each other, the pure channelrhodopsin kinetic can be extracted as shown in Fig. $3 \mathrm{c}$ and $3 \mathrm{f}$. The on, as well as the off, kinetic was fit exponentially as indicated in red and green respectively. The time constants of the channel kinetics in close proximity to 75 the stimulation point were extracted, and determined to be $11.3 \pm 0.2 \mathrm{~ms}$ for the on- and $34.5 \pm 0.2 \mathrm{~ms}$ for the offkinetics.

\section{Network analysis}

${ }_{80}$ Spreading of the APs could be monitored with the MEA and is shown in Fig. 4. If analyzed with respect to the origin of stimulation and the propagation direction the difference between intrinsic and artificial stimulation becomes clearer. The time bin of the action potential is color coded and plotted 85 for the $8 \times 8$ array of the MEA. In Fig. 4 a the data for the intrinsic pacemaker is shown. The origin of the intrinsic APs was on the right side of the active area and propagated over the MEA to the left. In contrast to the intrinsic propagation, the artificial, laser evoked, APs are shown in Fig. 4b. Here it 90 is visible that the artificial pacemaker, unlike the intrinsic one, is situated in the active area close to channel 55 as the signal starts from a point there and propagates in all directions. The black circles were included to facilitate visibility of the propagation.

${ }_{95}$ Fig. $5 \mathrm{~b}$ shows a single channel with both, subthreshold depolarization and triggered APs. Once an AP was triggered it 
a
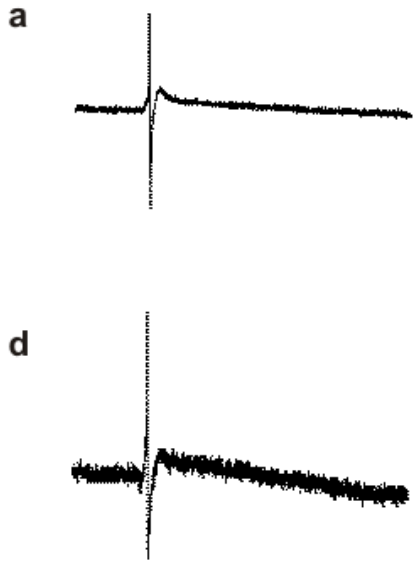

b

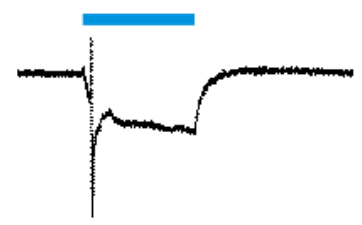

e

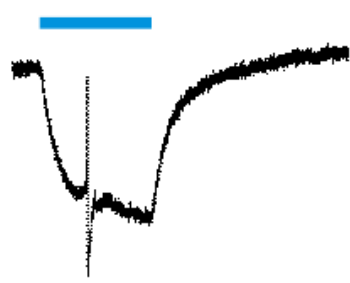

C

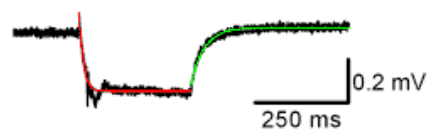

$\mathbf{f}$

Figure 3:

Two representative channels recording spontaneous and light evoked action potentials. Beats were extracted from different times during the experiment. a-c: a channel near the light stimulation. $d-f$ : a channel $200 \mu \mathrm{m}$ farther away from the light stimulation. The spontaneously occurring action 5 potential ( $a$ and $d$ ) is subtracted from the light induced AP ( $b$ and e) to extract the channelrhodopsin 2 kinetics ( $c$ and f). The resulting data is fit with an exponential for both the channel opening (red) kinetics and the channel closing (green) kinetics.

spread over the whole cell layer as visible in the full MEA recording. Cell connectivity over the whole array can be seen in Fig. 5c where AP events are plotted as a single point for all 10 channels during twenty seconds of recording. It can be seen that the sub-threshold stimulation test, where only depolarization was evoked, does not spread over the whole cell layer. Fig.5a shows the behavior of one segment of the array in detail. The recordings for channels near the 15 stimulation point (indicated by the red dot next to channel 55) are plotted in the geometry of the electrodes on chip. A single depolarization is highlighted by the blue box. The depolarization decreased with distance to the stimulation point and was not self activating like action potentials. Further 20 analysis was performed by plotting the ratio of the depolarization amplitude and the AP amplitude versus the distance from the origin (Fig.5d). Channel 55 was used as the origin because it was the closest recording to the stimulation location. The use of depolarization to AP ratio accounts for 25 differences in cell sensor coupling at each electrode. Besides changes in amplitude, the time course was also analyzed with respect to the distance from the stimulation point. The time constant for a channel $200 \mu \mathrm{m}$ away from the stimulation point, was $58.5 \pm .4$ and $111 \pm .7 \mathrm{~ms}$, for on- and off-kinetics 30 respectively. With increasing distance from the origin, the time constant increases and the signal visibly fades. In addition to the passive processes, the active AP propagation speed was analyzed in the form of a distance versus time shift plot and fit linearly (Fig.5e). The slope gives a propagation 35 speed of $0.61 \mathrm{~cm} / \mathrm{s}$. The speed of propagation for subthreshold depolarization was similar to that of AP propagation, though the small number of channels detecting subthreshold depolarization prevents more detailed analysis.

\section{Delay}

Besides experiments on evoking action potentials in the tissue, experiments concerning the refractory period were performed. For this reason the laser was manually triggered via a TTL pulse to flash directly after spontaneously occurring 45 APs. The recording setup was changed to allow recording of the TTL pulse in channel 1. Figure 6 shows the results of laser interference with beat rate. The recording of one channel and the respective TTL pulse for the laser are plotted versus the time. the TTL trace is always given in black. When laser ${ }_{50}$ flashes follow each action potential (Fig.6a) the inter-spike interval is extended from the spontaneous state of $3.0 \pm 0.5 \mathrm{~s}$ to $7.0 \pm 1.5 \mathrm{~s}$ (significance $\mathrm{a}<0.001$ (one-way Anova)) (Fig.6d). Intermittent interference (Fig.6b) results in a slightly longer overall inter-spike interval with individual spikes 55 following illumination more severely delayed. Upon termination of laser interference, the initial high beat frequency is resumed (Fig.6c). Figure 6d shows that with decreasing interference-speed the AP interval decreases, which is a rise in AP frequency. When laser pulses are 60 applied only occassionally after APs, the AP immediately after the light flash is most severely delayed (Fig.6b). Finally, 

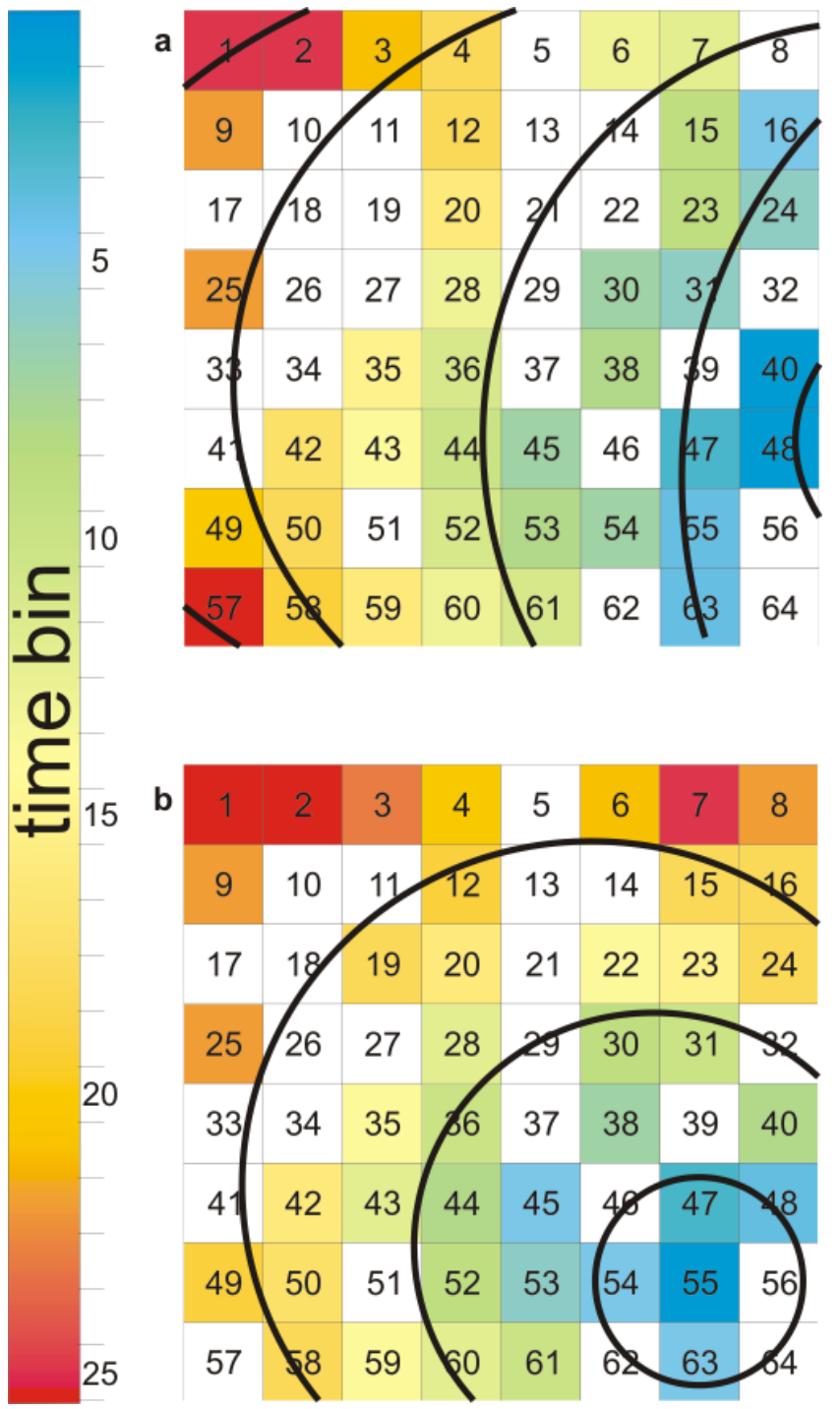

Figure 4: Signal propagation

Depiction of the channel layout on chip, numbers represent channel number. The bin for action potential events is color coded, as indicated 5 by the bar, from early (blue, 1) to late (red, 25). Binning was performed in $10 \mathrm{~ms}$ steps to facilitate analysis of the propagation direction.

A: $\quad$ shows the propagation for the intrinsic pacemaker. It can be seen that the signal arrives in a front at the right side of the chip and propagates from right to left.

10 B:

B: $\quad$ gives the propagation for the laser stimulation. The artificia pacemaker was situated between channel 55 and 56 and the signal propagates from there into all directions. Circles were included as a guide to the eye for the propagation direction.

without any influence from the laser, the constant, intrinsic 15 beat frequency is reestablished. These effects were seen across all channels of the chip (supplementary Fig. I).

\section{Discussion \& Outlook}

Bidirectionally interfacing cells is a promising approach in various fields such as prosthesis development and drug 20 screening. General problems, including non-damaging and reliable stimulation, have to be overcome for these interfaces
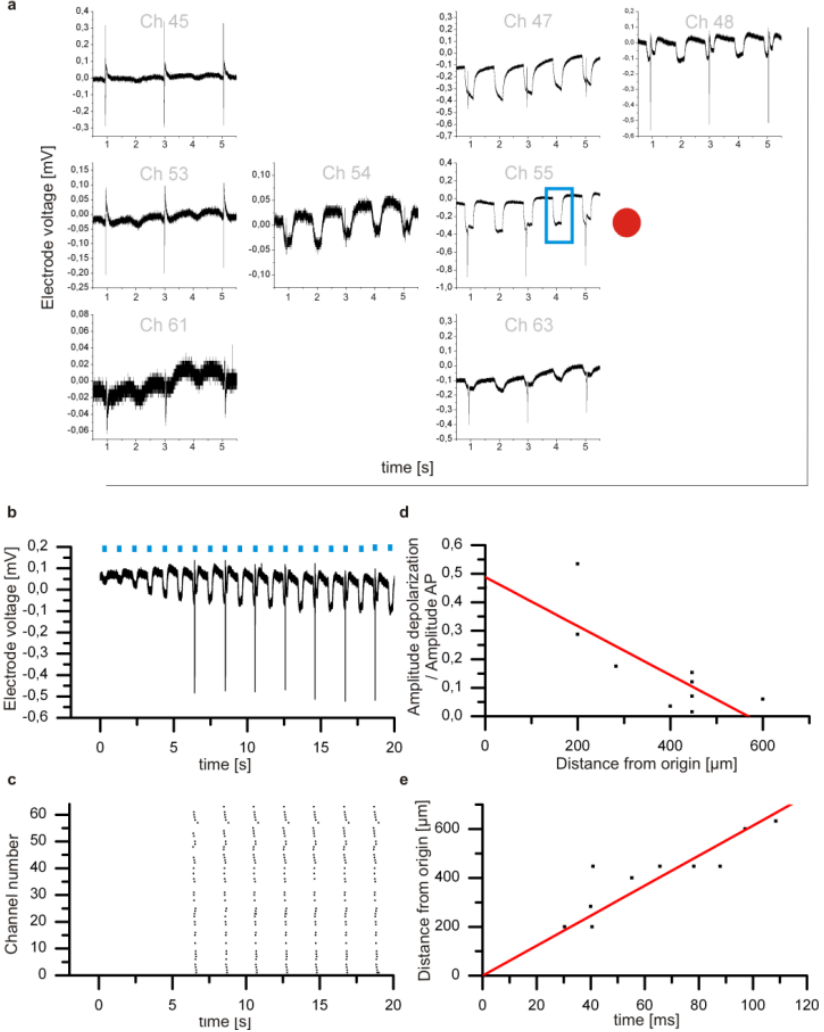

Figure 5: Stimulation effect on the network

A: $\quad$ The recorded channels of the lower right corner presented in 25 their original position on the MEA. On empty channels no signals were measured due to high noise or an electrode defect. Laser stimulation was performed between channel 55 and 56 as indicated by the red dot. The recorded depolarization without AP can be seen in the blue box. This depolarization decreases with distance and is no longer visible in channel 45 where only evoked APs arrive after spreading over the chip.

B: $\quad$ Trace of one channel. The laser pulses are indicated in blue. It can be seen that not every pulse triggers an AP but if an AP is evoked, the propagation spreads over all channels as shown in $\mathrm{C}$.

C: $\quad$ AP events of all 64 channels plotted versus the time.

D: $\quad$ The ratio of the depolarization amplitude / AP amplitude plotted versus the distance to the origin.

$40 \mathrm{E}$ :

Propagation of the action potential. The distance from the origin is plotted versus the time. The linear fit gives a slope of $0.61 \mathrm{~cm} / \mathrm{s}$.

to become the method of choice. In this work, we have shown that artifact free interfacing of HL-1 cells is possible using 45 channelrhodopsin. We introduced a stimulation setup that is independent of chip technology as it works with the microscope optics and is compatible with most microscopes. Furthermore, combined stimulation and extracellular measurements can be performed and were shown during this 50 study.

\section{Light stimulation}

HL-1 cardiomyocyte-like cells were employed as a model 

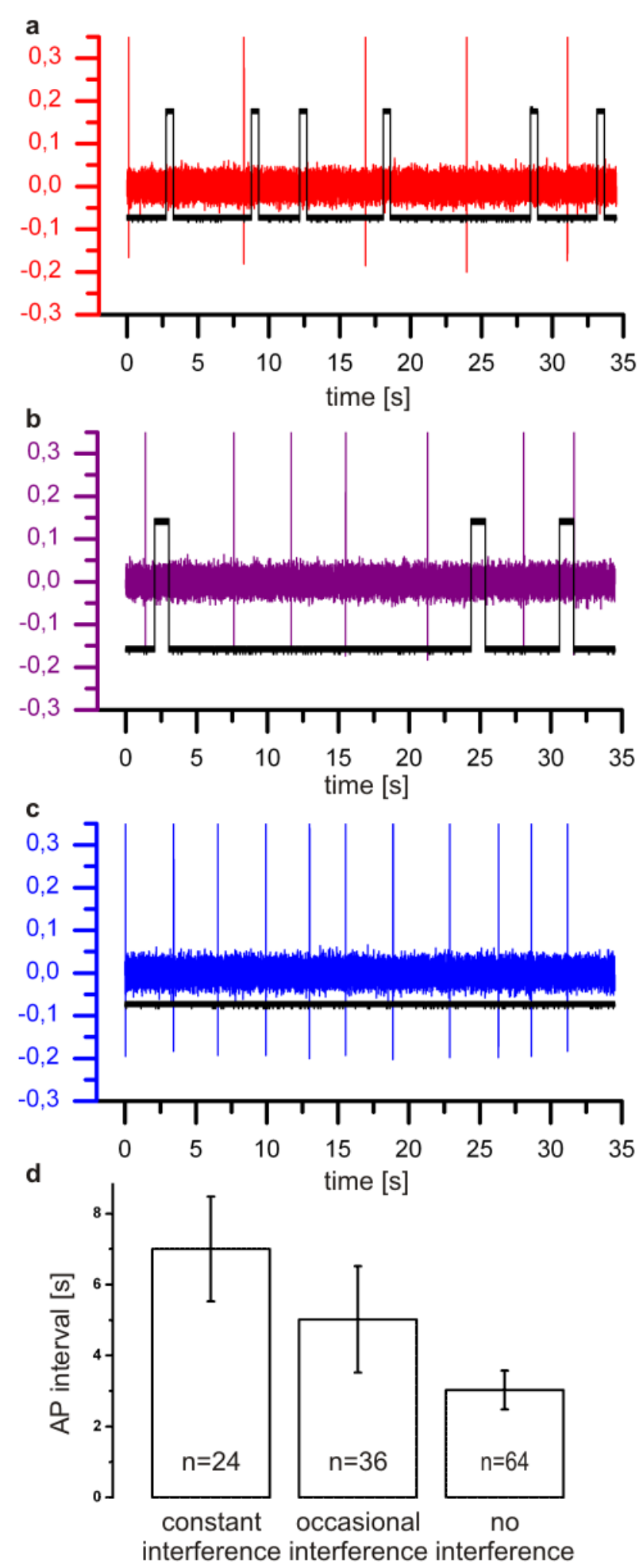

Figure 6: Light elongated refractory period (LERP).

APs respond to light evoked interference. Raw data is plotted above the TTL trace indicating laser activation.

$5 \mathrm{~A}: \quad$ Representative channel showing AP events when a laser flash follows each action potential. Inter-spike interval is strongly elongated.

B: $\quad$ Representative channel showing AP events when laser flashes occasionally disrupt intrinsic beating. Individual spikes are
D: Comparison of inter-spike intervals with constant interference, occassional interference, or no interference.

system for the beating heart. These cells formed confluent 20 layers on MEA arrays which were connected by gap junctions. If left for approximately one day after confluency, the cultures exhibit spontaneous beating, ie a wave of action potentials generated by an intrinsic pacemaker which propagate acroos the cell sheet. At full maturity, the AP wave is accompanied 25 by contraction that can be confirmed visually. We demonstrated the possibility of creating an artificial pacemaker cell in these cultures using light activatable channels. Channelrhodopsin and its light induced permeability to cations can be used to depolarize single transfected cells. 30 The incomplete transfection of the entire cell population, or plasmid loss during subsequent growth leads to a network of normal HL-1 cells with randomly dispersed Ch2 expressing cells. In only the YFP positive, and therefore $\mathrm{Ch} 2$ positive, cells, were we able to initiate depolarization with light. We 35 showed that this depolarization not only propagates through gap junctions over local areas of the tissue but can also evoke action potentials which spread over the whole tissue if the induced depolarization is above threshold. Furthermore, this effect was proven by analysis of the origin of stimulation and 40 the propagation direction of intrinsic and artificial light induced activity. An example measurement was shown where the intrinsic activation of the signal comes from the right side of the active area of the chip and propagates to the left, whereas the artificial pacemaker is situated in the lower right 45 corner of the active area and the signal spreads concentrically in all directions. This induced pacemaker switching might be applied in the future for further pacemaker analysis. Again the use of microelectrode arrays and their spatiotemporal resolution may enable investigations of pacemaker 50 interference patterns.

If analyzed in respect to single cells it was clearly shown that a light pulse depolarizes the cell and, upon reaching the threshold, evokes an action potential (Fig 2.c). The extracellular recording clearly consists of two different 55 kinetics. One the one hand, the action potential kinetics (Fig.3a and 3d) which consist of a fast upward spike that is related to the first membrane depolarization followed by the opening of sodium channels and a fast inward sodium current that correlates to the fast downward spike. The shape of the 60 extracellularly recorded action potential is further explained in Fig.1. On the other hand, the light evoked signal consists of the channelrhodopsin induced kinetic (Fig.2d). Opening of the channels in the attached membrane over the electrode triggers an influx of positive ions, which spread over the cell layer, 65 maintaining a gradient and a constant current over the cell membrane. As the current flows constantly the whole time the channels stay open, the extracellular recorded electrode 
voltage stays at a constant level. After closing of the channel the cleft recovers the initial ion composition and the voltage goes back to zero. The negative sign of the extracellular signal is due to the position of the electrode. As the cleft between the 5 cell and the electrode is depleted of positive ions, a negative deflection of the signal is observed.

There are discrepancies in the literature regarding normal channel kinetics for wild type channelrhodopsin. $\tau_{\text {on }}$ and $\tau_{\text {off }}$ calculated here for $\mathrm{ChR} 2$ kinetics are expected to vary from that 10 reported in the literature due to the nature of the measurement. Initial simulation of the on and off kinetics used giant membrane patches to estimate $\tau_{\text {on }}$ of $.2 \mathrm{~ms}$ and $\tau_{\text {off }}$ of $20 \mathrm{~ms}$ [32]. More recent current clamp measurements of whole cells report $\tau_{\text {on }}$ of ChR2 to be $55.5 \mathrm{~ms}$ while $\tau_{\text {off }}$ is $9.8 \mathrm{~ms}$ [45]. However, in both of 15 these cases $\tau_{\text {on }}$ was determined from channel opening to the establishment of steady-state current, including the closing of a fraction of the channels in the continuously illuminated state. In our voltage measurements, $\tau_{\text {on }}$ is determined by an exponential fit from the start of deoplarization, through the steady state. In the 20 case of $\tau_{\text {off, }}$ patch clamp experiments experience a direct cessation of ion flow when the channels in the membrane are closed. In contrast, when using MEA recording, the ion concentration in the gap between the cell and electrode returns to a neutral charge compared to the bath after channels in the cell 25 close via diffusion of ions between the cell and the substrate. The higher this leak resistance is, the better cell-electrode coupling is for signal detection, however high leak resistance will also delay recovery of the electrode to baseline if a large concentration difference has developed between the gap and the bath during 30 prolonged channel opening.

In an immature culture that has formed gap junctions but has not started spontaneously beating, it can be seen that in most cases every second light stimulation resulted in an action potential that spreads over the cell layer (Fig.4a-c). This is 35 due to the second pulse arriving while the cell is still in the relative refractory period, where the AP excitation is not possible under the same conditions but requires a higher initial depolarization. In this scenario, if only two light stimulation pulses were applied to the culture, only a single 40 AP would be produced. This is in contrast to AP delay in a spontaneously beating culture, where cessation of light pulses would result in a return to the spontaneous beat rate.

If analyzed in respect to the network activity, we showed that the light triggered APs spread over the tissue and behave 45 normally. The propagation speed of the action potential is not different from the intrinsic one or reference measurements. Besides the evoked APs, the locally restricted channelrhodopsin induced depolarization can be analyzed. For better comparison between the channels, the ratio of the s0 depolarization and the respective action potential was calculated in order to normalize the measured values. An AP follows an all or nothing reaction with constant change in membrane potential and thus can be used for normalization of the measured voltage on different electrodes as absolute signal 55 varies with performance of the electrode and electrical coupling. Two effects can be observed qualitatively. On the one hand, the normalized depolarization recorded versus the distance gives information on the attenuation of the signal. Therefore, attenuation over distance can be observed. On the 60 other hand, a change in the time course can be seen. The time constant tau of the channelrhodopsin kinetic (Fig.2d.) gets bigger with increasing distance to the origin. This can be explained if the cell layer is analyzed in respect to its electrical properties. The cells separated by gap junctions ${ }_{65}$ behave like a resistor in series with a capacitor. A signal that propagates over the cell layer is, due to these properties, low pass filtered (RC-filter), resulting in the observed fading of the signal. This finding is in good agreement with the cable conduction theory, which models the signal propagation 70 through a cable and other cell types e.g. a neuronal axon.

\section{Delay}

Furthermore, we demonstrated that not only the activation of APs, but also suppression of them is possible, as was shown in the elongated refractory period for light pulses applied 75 directly after an action potential. This light elongated refractory period (LERP) could be introduced with a single laser flash and in darkness cells recovered directly to their original behavior. No damping effect could be observed over time. The mechanism of the delay can be explained if one 80 considers a standard myocyte AP. The initial state is the resting potential. Depolarization happens due to electrodiffusion of ions from neighboring cells that already fire an action potential or experience a sub-threshold depolarization (Fig.1c,d, orange). When these ions depolarize 85 the cell to threshold, sodium permeability increases as voltage gated channels open and the cell is further depolarized (Fig.1c,d green). Closing of the channels results in a slight repolarization followed by a plateau which is governed by a $\mathrm{Ca}^{2+}$ influx in equilibrium with a $\mathrm{K}^{+}$efflux (not visible in ${ }_{90}$ Fig.1c). The duration of the plateau determines the refractory period, during which the cell will not generate an AP in response to ions diffusing across the gap junctions. Declining calcium permeability results in repolarization to the resting potential and regained ability to fire APs.

95 In artificial cell pacing, light induced opening of channelrhodopsin results in an influx of positive charge into the cell and subsequent depolarization. Diffusion through gap junctions affects neighboring cells also. If this depolarizing pulse is above the threshold of voltage gated $\mathrm{Na}^{+}$channels 100 they become activated, resulting in an AP. However, if the light pulse is given during the plateau phase of the AP, the channelrhodopsin induced influx of positive charges can compensate for the potassium efflux. This results in an elongated plateau phase and an increase of the refractory 105 period where no further AP can be elicited. This delay of action potentials is possible in two ways. Firstly, if the affected area is not in proximity to the intrinsic pacemaker, the AP suppression is only local and restricted to the area of depolarization. Secondly, as demonstrated in this study, the 110 stimulation may affect the original pacemaker and prevent generation of APs. Normally, the pacemaker cell depolarizes automatically and fires intrinsic APs, but if the channelrhodopsin is activated after depolarization, the interspike-interval increases in the pacemaker cell and results 115 in the global suppression of APs. If the suppression is too long, other, slower pacemakers from a more distant position can take over. 


\section{Application}

The technique shown in this study may be applicable to confocal microscopy techniques as well. In studies where 5 movement interferes with optical measurements, techniques are sought to inhibit myocyte contraction with minimal perturbation of the cell. For example, Pelloux et al. study pacemaker free cultures. The optical control of confocal microscopy lasers would allow pulses of various intensities of 10 light to be applied in a suppression regime interspersed with optical measurements. Laser stimulation could conversely be used to activate model systems that do not normally produce reliable APs [46].

Furthermore, greater control over individual regions of tissue 15 allows specific in vitro studies of cardiac function. Mixed cell type cultures or physical barriers are already used to investigate conduction through non-homogeneous cardiac tissue [47, 48]. Optical intervention with ChR2 allows individual cells to be manipulated for creating interference 20 patterns in natural conduction or to mimic the partial depolarization experienced by cardiomyocytes when gap junctions form with non-cardiomyocyte cells.

However, not only in vitro studies can take advantage of this technique. Current medical technology is analyzing new ways 25 of interfacing electrogenic cells. Our technique now gives access to bidirectionally interfacing electrogenic cells without the drawbacks of electrical stimulation. For stimulation of tissue the electrode stimulation needs to be charge balanced, which is not a limitation for ChR2. Furthermore, 30 permeabilisation of cells is an issue of electric stimulation, whereas ChR2 gives the advantage of artifact free and reliable stimulation without permeabilization. Nevertheless, electric pacemakers are commonly used and well explored. ChR2 would not be an immediate alternative as transfection 35 techniques need to be found and implants (not for electrical stimulation but to provide a light source to the cells) would still be necessary. However, the application of defibrillating cells makes investigation of channelrhodopsin in cardiac cells an interesting pursuit. The use of channelrhodopsin not only 40 allows us to trigger action potentials but also to prevent them based on the phase of the action potential perturbed. One imaginable device is a combined cardiac pacemaker and defibrillator. On the one hand it could be used as a pacemaker by generating APs, and on the other hand it could be used for 45 reducing the amount of APs and thereby suppressing fibrillation. The required light intensities could also be provided by state of the art LEDs [49] allowing the generation of an implantable medical device [43].

\section{Experimental}

\section{${ }_{50}$ Recording setup}

We used a custom, 64-channel amplifier system with microelectrodes coupled directly to the inputs of highimpedance operational amplifiers (preamplifier gain 10.22), then coupled to a main amplifier (gain 100) providing an 55 overall gain of 1022 . The high-pass performance depended only on the size, material, surface condition, and cleanliness of the microelectrodes, providing a large bandwidth recording system. This high-impedance input provides a bandwidth of $0.4 \mathrm{~Hz}-3.9 \mathrm{kHz}$ for electrodes with a diameter of $20 \mu \mathrm{m}$ in our ${ }_{60}$ MEA system. This performance enables reliable recordings of distinct signal shapes of extracellularly recorded action potentials (APs) originating from individual cells. Most importantly, recordings with our setup can be done with cheap-to-fabricate, reusable, planar gold microelectrodes, with ${ }_{65}$ sizes smaller than the soma of individual cells. Data were sampled simultaneously across all channels at $10 \mathrm{kHz}$ per channel using the MED64 Conductor 3.1 software (Alpha MED Sciences, Japan). An extracellular $\mathrm{Ag} / \mathrm{AgCl}$ electrode, set to ground potential, served as a reference electrode. A 70 more detailed description of the data acquisition has been published previously $[50,51]$.

\section{Microelectrode arrays}

The MEAs were manufactured on glass wafers using standard silicon technology. The planar 64 -channel gold MEAs $(8 \times 8)$ 75 were designed with diameters of $6,8,10$ or $20 \mu \mathrm{m}$ at a pitch of 100 or $200 \mu \mathrm{m}$. Chips were passivated by an oxide-nitrideoxide layer deposited by plasma-enhanced chemical vapor deposition consisting of $500 \mathrm{~nm} \mathrm{SiO}_{2}, 500 \mathrm{~nm} \mathrm{Si} 3 \mathrm{~N}_{4}$, and 100 $\mathrm{nm} \mathrm{SiO}_{2}$. Details of the fabrication and encapsulation have 80 been described previously [50, 51].

\section{Laser stimulation}

Activation of ChR2 was achieved using a $100 \mathrm{~mW} 473 \mathrm{~nm}$ diode laser (Rapp OptoElectronic GmbH Hamburg, Germany). The laser was coupled to the optical path of the microscope 85 via an optical fiber and reflected into the light path by a dichroic mirror (UGA-40, Rapp OptoElectronic $\mathrm{GmbH}$ Hamburg, Germany). Epifluorescent and brightfield illumination was possible via additional light sources. The spot diameter of the laser is adjustable with the fiber, 90 objective and focus, and was set to about $10 \mu \mathrm{m}$ during the experiments. The laser was controlled by TTL pulses from the laser control unit or software. Minimum stimulation time was $1 \mathrm{~ms}$ and maximum power about $1000 \mathrm{~mW} / \mathrm{mm}^{2}$. A power of around $10 \mathrm{~mW} / \mathrm{mm}^{2}$ proved sufficient to activate ${ }_{95}$ Channelrhodopsin 2 [35].

\section{Channelrhodopsin/Plasmid}

The algal rhodopsin channelrhodopsin 2 (Ch2) was identified in $C$. reinhardtii [32] and a mammalian transient expression vector for the protein has been generated [33]. The leading 100309 amino acids (AA) of Ch2 were fused to eYFP for a final 553 AA protein. Addition of the EYFP tail was previously shown not to interfere with channel function [33]. Retinal in mammalian cell cultures binds covalently to the expressed $\mathrm{Ch} 2$ to form ChR2. Blue light triggered retinal switching from 105 the trans to cis state results in the conformational change of ChR2 allowing cations to flow. Thus, unlike G-protein coupled vertebrate rhodopsins, ChR2 provides a directly light gated ion channel.

Cells were transfected with either the CH2-K315-YFP 110 plasmid (kind gift from the Bamberg group) or a modified 
CH2-K315-YFP plasmid containing the human Ubiquitin $\mathrm{C}$ promoter from Matsuda \& Cepko 104 bases ahead of the Ch2 coding sequence, plasmid CMV_Ubi_Ch2_YFP.

\section{Cell culture and transfection}

5 The HL-1 cell line (Louisiana State University Health Science Center, New Orleans, LA, USA), was derived from AT-1 cells (mouse cardiomyocyte tumor). It represents a hybrid between embryonic and adult myocytes [52]. The HL-1 cardiac muscle cells show spontaneous APs and subsequent contraction after 10 the cells reach confluency. Cells were cultured in T25 flasks at $37^{\circ} \mathrm{C}$ and $5 \% \mathrm{CO}^{2}$ in Claycomb Medium with $10 \% \mathrm{FBS}$, $100 \mu \mathrm{g} / \mathrm{ml}$ Penicilin-Streptomycin, $0.1 \mathrm{mM}$ Norepinephrine and $2 \mathrm{mM}$ L-Glutamine in a humidified chamber. After cells reached confluency and started beating, they were seeded 15 onto the chips as described by Law et al. [53]. Briefly, cells were rinsed with PBS then trypsinized using $1 \mathrm{ml} 0.05 \%$ trypsin/EDTA. Trypsinization was stopped by adding $5 \mathrm{ml}$ of medium followed by centrifugation $\left(\begin{array}{llll}5 & \min & 500 & \mathrm{~g}\end{array}\right)$. Supernatant was removed and the pellet was resuspended in ${ }_{20} 0.1 \mathrm{ml}$ Transfection Solution (Lonza) and 3-5 $\mu \mathrm{g}$ of plasmid (see above). Transfection was performed using the AMAXA nucleofector device in the associated cuvettes. Afterwards, the cell solution was diluted with $0.5 \mathrm{ml}$ of supplemented Claycomb Medium. After counting the cells, approximately ${ }_{25} 3000$ cells $/ \mathrm{mm}^{2}$ were plated in $50 \mu \mathrm{l}$ of medium on 64 electrode MEAs coated with gelatin fibronectin. After 4 hours of adhesion, chips were filled with $500 \mu \mathrm{l}$ of medium. Medium was changed daily. Measurements were performed after cells reached confluency, usually after 2-4 days in vitro. ${ }_{30}$ Furthermore, some experiments used the FuGene HD kit (Roche) for on chip transfection according to the manufacturer's protocol.

\section{Data analysis}

AP time stamps were extracted using the Plexon Offline ${ }_{35}$ Sorter (Plexon inc.) and further analysed using the Neuroexplorer4 (NexTechnologies) and Origin. For analysis of the AP propagation over the chip, time bins of $10 \mathrm{~ms}$ were color coded and assigned to the electrode with a detected AP.

\section{Acknowledgements}

${ }_{40}$ We gratefully acknowledge the help of Rita Fricke, especially in the cell culture. Marko Banzet and Michael Prömpers for processing of the wafers, and all other members of the IBN-2 for fruitful discussions and an excellent working atmosphere. We thank Prof. W. Claycomb for the kind gift of HL-1 cells.

${ }_{45}$ Furthermore, Vanessa Maybeck acknowledges financial support by the International Helmholtz Research School on Biophysics and Soft Matter ("BioSoft").

\section{Notes and references}

${ }^{a}$ Institute of Bio- and Nanosystems-Bioelectronics (IBN-2) and Jara50 FIT, Leo-Brandt-Str., Forschungszentrum Jülich, D-52425 Jülich, Germany

${ }^{b}$ Department of Informatics and Microsystem Technology, University of Applied Sciences Kaiserslautern, D-66482 Zweibrücken, Germany
${ }^{c}$ Max-Planck-Institute of Biophysics, Max-von-Laue Strasse 3, D-60438 55 Frankfurt, Germany; and Institut für Biophysikalische Chemie, Johann

Wolfgang Goethe University, Max-von-Laue-Strasse 9, D-60438

Frankfurt, Germany

${ }^{*}$ Prof. Dr. Andreas Offenhäusser, Leo-Brandt-Str., Forschungszentrum

Jülich, D-52425 Jülich, Germany, Tel: 00492461 612330, Fax: 0049

602461 618733,e-mail:a.offenhaeusser@fz-juelich.de

" These authors contributed equally

$\dagger$ Electronic Supplementary Information (ESI) available: [See end of document]. See DOI: 10.1039/b000000x/

1 G. M. Clark, Philosophical Transactions Of The Royal Society BBiological Sciences, 2006, 361, 791-810;

2 J. H. Ye and Y. S. Goo, Conf Proc IEEE Eng Med Biol Soc, 2007, 2007, 5783-5786;

703 E.-T. Kim, C. Kim, S.-W. Lee, J.-M. Seo, H. Chung and S.-J. Kim, Invest Ophthalmol Vis Sci, 2009;

4 S. Micera, X. Navarro, J. Carpaneto, L. Citi, O. Tonet, P. M. Rossini, M. C. Carrozza, K. P. Hoffmann, M. Vivó, K. Yoshida and P. Dario, IEEE Trans Neural Syst Rehabil Eng, 2008, 16, 453-472;

755 X. Navarro, T. B. Krueger, N. Lago, S. Micera, T. Stieglitz and P. Dario, J Peripher Nerv Syst, 2005, 10, 229-258;

6 J. K. Chapin, Nat Neurosci, 2004, 7, 452-455

7 A Stett, U Egert, E Guenther, F Hofmann, T Meyer, W Nisch, and H Haemmerle, Anal Bioanal Chem, 2003, 377(3), 486-495;

808 M. Reppel, F. Pillekamp, Z. J. Lu, M. Halbach, K. Brockmeier, B. K. Fleischmann and J. Hescheler, J Electrocardiol, 2004, 37 Suppl, 104109 ;

9 C. K. Yeung, F. Sommerhage, G. Wrobel, A. Offenhäusser, M. Chan and S. Ingebrandt, Anal Bioanal Chem, 2007, 387, 2673-2680

8510 M. Chiappalone, A. Vato, L. Berdondini, M. Koudelka-Hep and S. Martinoia, Int J Neural Syst, 2007, 17, 87-103;

11 F. Heer, W. Franks, A. Blau, S. Taschini, C. Ziegler, A. Hierlemann and H. Baltes, Biosens Bioelectron, 2004, 20, 358-366;

12 U Frey, U Egert, F Heer, S Hafizovic, and A Hierlemann, Biosens $90 \quad$ Bioelectron, 2009, 24(7), 2191-2198;

13 I. Schoen and P. Fromherz, Biophys J, 2007, 92, 1096-1111;

14 D. A. Wagenaar and S. M. Potter, J Neurosci Methods, 2002, 120, 113-120;

15 D. A. Wagenaar and S. M. Potter, J Neural Eng, 2004, 1, 39-45

9516 Y. T. Wong, N. Dommel, P. Preston, L. E. Hallum, T. Lehmann, N. H. Lovell and G. J. Suaning, Ieee Transactions On Neural Systems And Rehabilitation Engineering, 2007, 15, 425-434;

17 A. Y. Chow and V. Y. Chow, Neuroscience Letters, 1997, 225, $13-$ 16 ;

10018 D. B. McCreery, Hearing Research, 2008, 242, 64-73;

19 M. Jahanshahi, C. M. A. Ardouin, R. G. Brown, J. C. Rothwell, J. Obeso, A. Albanese, M. C. Rodriguez-Oroz, E. Moro, A. L. Benabid, P. Pollak and P. Limousin-Dowsey, Brain, 2000, 123, 1142-1154;

20 C.-T. Tsai, L.-P. Lai, J.-J. Hwang, W.-P. Chen, F.-T. Chiang, K.-L.

105 Hsu, C.-D. Tseng, Y.-Z, Tseng, and J.-L. Lin, J Hypertens, 2008, 26(3), 570-582;

21 C.T. Tsai, F.-T. Chiang, C.-D. Tseng, J.-J. Hwang, K.-T Kuo, C.-K. Wu, C.-C Yu, Y.-C. Wang, L.-P. Lai, and J.-L. Lin, J Am Coll Cardiol, 2010, 55(8), 758-770;

11022 K. Jeffrey and V. Parsonnet, Circulation, 1998, 97, 1978-1991;

23 M. Zoll, New England Journal Of Medicine, 1952, 247, 768-771;

24 A. S. Manolis, H. Rastegar and N. A. Estes, JAMA, 1989, 262, 1362-1368;

25 R. W. Griffith and D. R. Humphrey, Neuroscience Letters, 2006, 406, 81-86;

26 D. B. McCreery, W. F. Agnew, T. G. Yuen and L. Bullara, IEEE Trans Biomed Eng, 1990, 37, 996-1001;

27 D. Ghezzi, A. Menegon, A. Pedrocchi, F. Valtorta and G. Ferrigno, J Neurosci Methods, 2008, 175, 70-78;

12028 P. Gorostiza and E. Y. Isacoff, Science, 2008, 322, 395-399;

29 S. Herlitze and L. T. Landmesser, Curr Opin Neurobiol, 2007, 17, 87-94;

30 R. D. Airan, K. R. Thompson, L. E. Fenno, H. Bernstein and K. Deisseroth, Nature, 2009, 458, 1025-1029; 
31 A. Berndt, O. Yizhar, L. A. Gunaydin, P. Hegemann and K. Deisseroth, Nat Neurosci, 2009, 12, 229-234;

32 G. Nagel, T. Szellas, W. Huhn, S. Kateriya, N. Adeishvili, P. Berthold, D. Ollig, P. Hegemann and E. Bamberg, Proc Natl Acad Sci U S A, 2003, 100, 13940-13945;

33 G. Nagel, T. Szellas, S. Kateriya, N. Adeishvili, P. Hegemann and E. Bamberg, Biochem Soc Trans, 2005, 33, 863-866;

34 F. Zhang, A. M. Aravanis, A. Adamantidis, L. de Lecea and K. Deisseroth, Nat Rev Neurosci, 2007, 8, 577-581;

1035 E. S. Boyden, F. Zhang, E. Bamberg, G. Nagel and K. Deisseroth, Nat Neurosci, 2005, 8, 1263-1268;

36 M. J. Wright, L. M. L. Wightman, D. S. Latchman and M. S. Marber, Gene Therapy, 2001, 8, 1833-1839;

37 C. Trollet, D. Scherman and P. Bigey, Methods Mol Biol, 2008, 423, $15 \quad$ 199-214;

38 J. D. Schertzer and G. S. Lynch, Methods Mol Biol, 2008, 433, 115125 ;

39 T. Inoue and R. Krumlauf, Nat Neurosci, 2001, 4 Suppl, 1156-1158;

40 P. S. Lagali, D. Balya, G. B. Awatramani, T. A. Munch, D. S. Kim,

20 V. Busskamp, C. L. Cepko and B. Roska, Nature Neuroscience, 2008, 11, 667-675;

41 A. D. Douglass, S. Kraves, K. Deisseroth, A. F. Schier and F. Engert, Current Biology, 2008, 18, 1133-1137;

42 A. M. Aravanis, L. P. Wang, F. Zhang, L. A. Meltzer, M. Z. Mogri,

25 M. B. Schneider and K. Deisseroth, Journal Of Neural Engineering, 2007, 4, S143-S156;

43 J. G. Bernstein, X. Han, M. A. Henninger, E. Y. Ko, X. Qian, G. T. Franzesi, J. P. McConnell, P. Stern, R. Desimone and E. S. Boyden, Proc Soc Photo Opt Instrum Eng, 2008, 6854, 68540H;

3044 J.P. Fahrenbach, X Ai, and K Banach, J Mol Cell Cardiol, 2008, 45(5), 642-649;

45 L.A. Gunaydin, O Yizhar, A Berndt, V.S. Sohal, K Deisseroth, and P Hegemann, Nat Neurosci, 2010, 13(3), 387-392;

46 S. Pelloux, J. Robillard, R. Ferrera, A. Bilbaut, C. Ojeda, V. Saks, M.

35 Ovize and Y. Tourneur, Prog Biophys Mol Biol, 2006, 90, 270-298;

47 S Rohr, J.P. Kucera, and A.G. Kleber, News In Physiological Sciences, 1997, 12, 171-177;

48 S. Rohr, Heart Rhythm, 2009, 6(6), 848-856;

49 L. Campagnola, H. Wang and M. J. Zylka, Journal of Neuroscience

40 Methods, 2008, 169, $27-33$;

50 H. Ecken, S. Ingebrandt, M. Krause, D. Richter, M. Hara and A. Offenhausser, Electrochim. Acta, 2003, 48, 3355-3362;

51 M. Krause, S. Ingebrandt, D. Richter, M. Denyer, M. Scholl, C. Sprössler and A. Offenhäusser, Sensors and Actuators B: Chemical, 2000, 70, 101 - 107;

52 W. C. Claycomb, N. A. Lanson, B. S. Stallworth, D. B. Egeland, J. B. Delcarpio, A. Bahinski and N. J. Izzo, Proc Natl Acad Sci U S A, 1998, 95, 2979-2984;

53 J. K. Y. Law, C. K. Yeung, B. Hofmann, S. Ingebrandt, J. A. Rudd,

50 A. Offenhäusser and M. Chan, Physiol Meas, 2009, 30, 155-167; 


\section{Supplementary information}

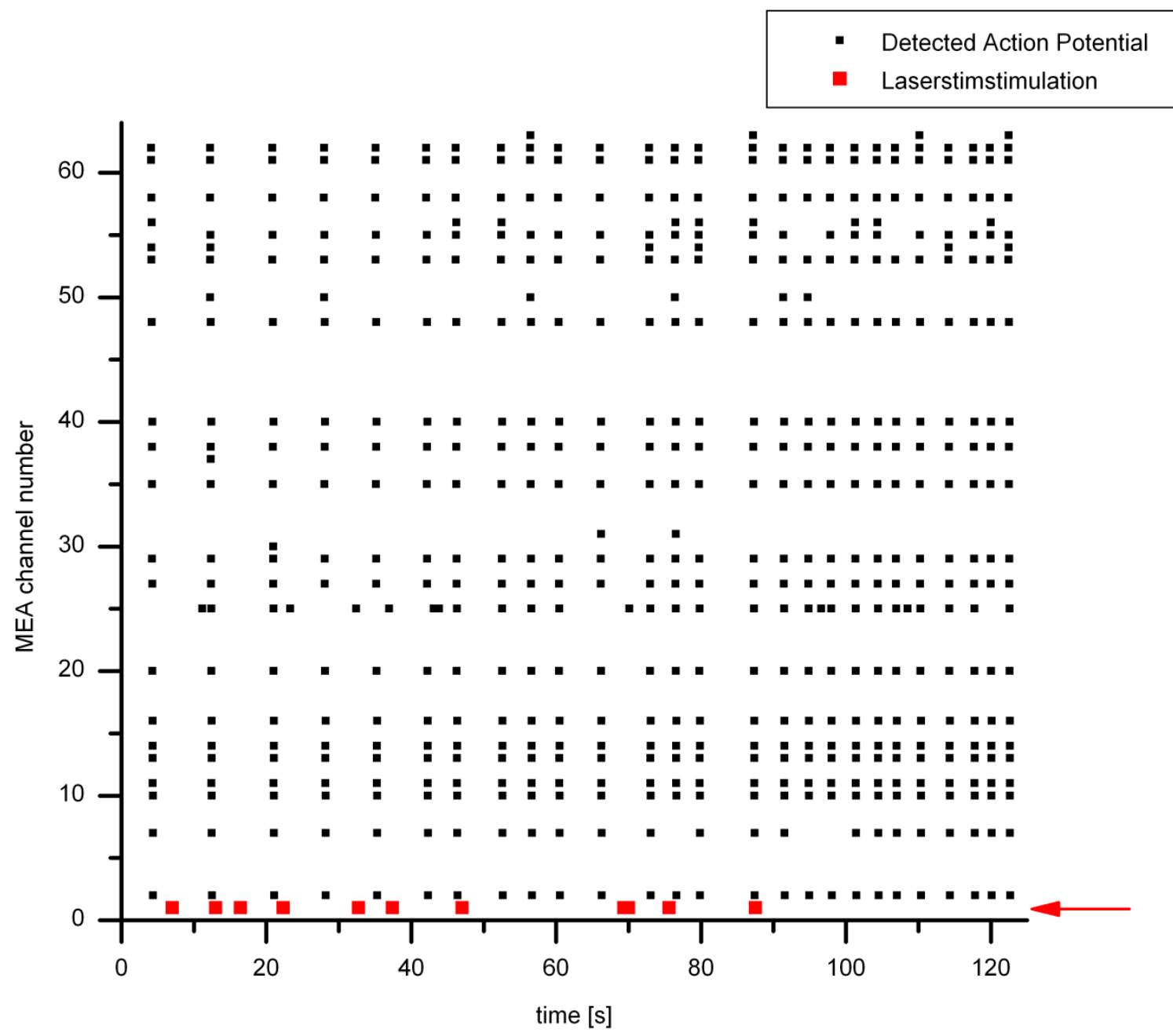

5 Sup I: $\quad$ AP event vs. time for light increased refractory period. On channel 1 (indicated by arrow) the timestamp of the stimulation can be seen (red). Stimulation was timed to occur after the APs. In the beginning of the recording every AP was followed by a light pulse, during the middle of the recording only a few flashes were administered and at the end no light flashes were applied. 